\title{
OBJECTS FOR CONCURRENT CONSTRAINT PROGRAMMING
}


THE KLUWER INTERNATIONAL SERIES IN ENGINEERING AND COMPUTER SCIENCE 


\title{
OBJECTS FOR CONCURRENT CONSTRAINT PROGRAMMING
}

\author{
by
}

Martin Henz

National University of Singapore

Singapore

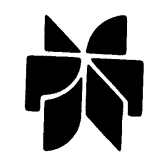

SPRINGER SCIENCE+BUSINESS MEDIA, LLC 
ISBN 978-1-4613-7502-9 ISBN 978-1-4615-5477-6 (eBook)

DOI 10.1007/978-1-4615-5477-6

\section{Library of Congress Cataloging-in-Publication Data}

A C.I.P. Catalogue record for this book is available from the Library of Congress.

Copyright (C) 1998 by Springer Science+Business Media New York Originally published by Kluwer Academic Publishers in 1998 Softcover reprint of the hardcover 1st edition 1998

All rights reserved. No part of this publication may be reproduced, stored in a retrieval system or transmitted in any form or by any means, mechanical, photocopying, recording, or otherwise, without the prior written permission of the publisher, Springer Science+Business Media, LLC.

Printed on acid-free paper. 
TO KELLY 


\section{CONTENTS}

FOREWORD BY GERT SMOLKA

xvii

PREFACE

xix

ACKNOWLEDGEMENTS

$\mathbf{x x i}$

Part I SETTING THE STAGE 1

1 INTRODUCTION 3

1.1 Area of Research . . . . . . . . . . . . . . . 3

1.2 Programming Language Design $\ldots \ldots \ldots \ldots \ldots$

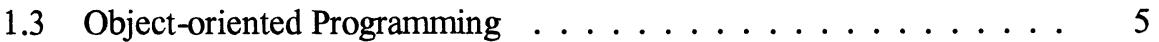

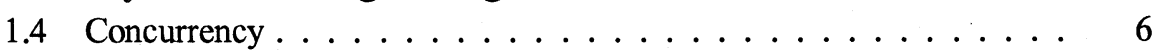

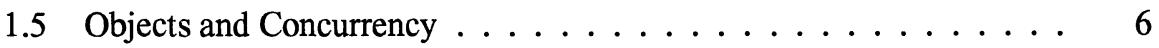

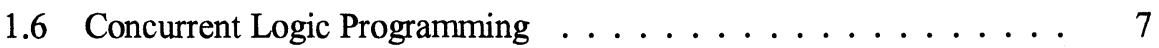

1.7 The Language $\mathrm{Oz} \ldots \ldots \ldots \ldots \ldots$

1.8 The Development of Objects in $\mathrm{Oz} \ldots \ldots \ldots \ldots$

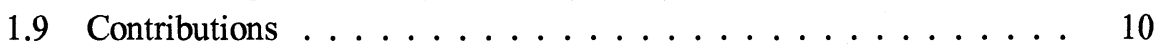

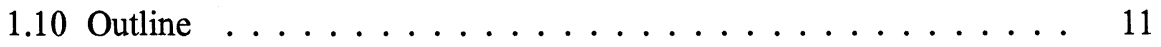

References . . . . . . . . . . . . . . . . . 13

2 ISSUES IN OBJECT-ORIENTED LANGUAGE DESIGN 17

2.1 Knowledge Representation View . . . . . . . . . . . . . . . 17

2.2 Software Development View . . . . . . . . . . . . . . 18

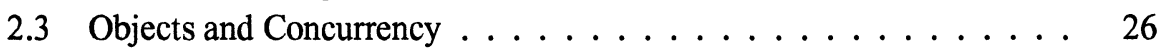

2.4 Further Issues $\ldots \ldots \ldots \ldots \ldots \ldots \ldots \ldots$

2.5 Historical Notes . . . . . . . . . . . . . . . . . . . . . . 29

References . . . . . . . . . . . . . . . . . . . . . . 29 
3 SMALL OZ 35

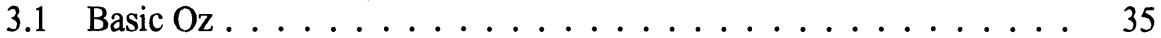

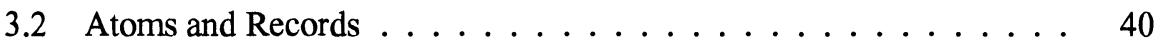

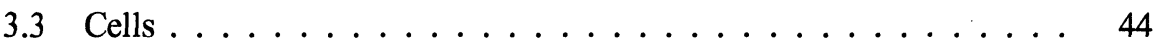

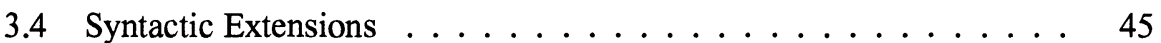

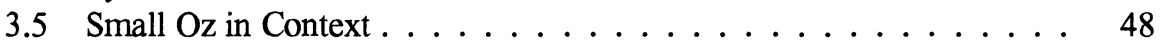

References ..................... 50

4 FIRST STEPS TOWARDS OBJECTS 53

4.1 Objects in Functional Programming with State . . . . . . . . . . 53

4.2 Objects in Concurrent Logic Programming . . . . . . . . . . . . . 57

References ...................... 60

\section{Part II OBJECT-ORIENTED PROGRAMMING 61}

5 BASIC OBJECT SYSTEM 63

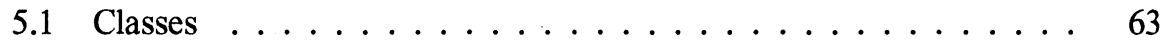

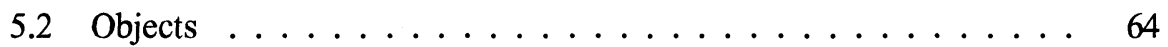

5.3 Inheritance for Conservative Extension . . . . . . . . . 65

5.4 Inheritance for Non-Conservative Extension . . . . . . . . . . 66

5.5 Case Study: Sieve of Eratosthenes ............... 66

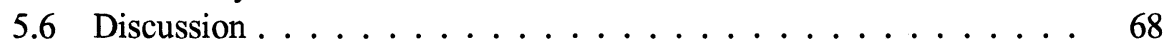

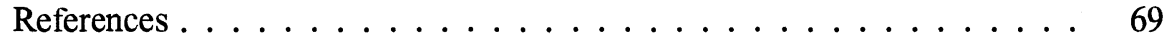

6 ADVANCED TECHNIQUES

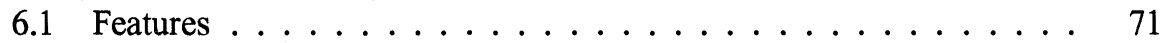

6.2 Free Attributes and Features . . . . . . . . . . . . . . 72

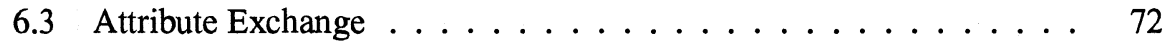

6.4 Multiple Inheritance . . . . . . . . . . . . . . . . 73

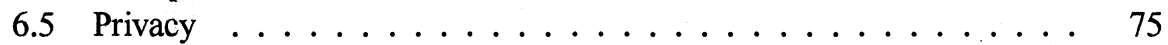

6.6 First-Class Messages and Message Patterns . . . . . . . . . 78

6.7 Higher-Order Programming with State . . . . . . . . . . . . 79

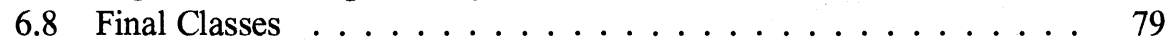

6.9 Classes as First-Class Values . . . . . . . . . . . . . . . . 80

6.10 First Class Attribute Identifiers . . . . . . . . . . . . . . . . . . . . . . . . . . . 81

6.11 Case Study: N-Queens . . . . . . . . . . . . . . . . . 82

6.12 Discussion . . . . . . . . . . . . . . 85

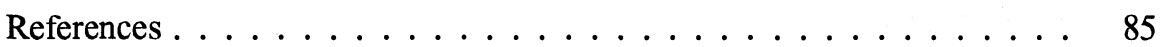

7 REDUCTION TO SMALL OZ 87

7.1 Class Definition . . . . . . . . . . . . . . . . . 87

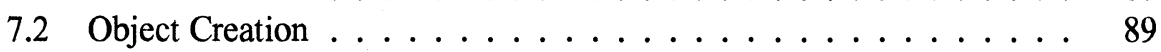

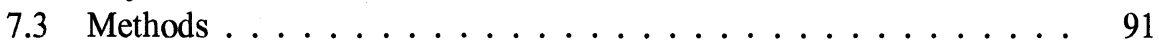

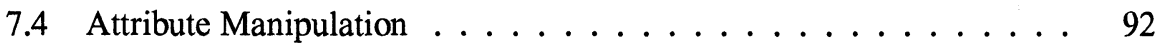

7.5 Object and Method Application . . . . . . . . . . . . . 92 


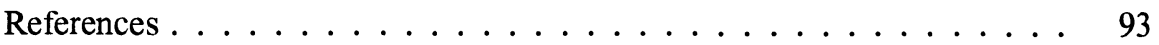

8 IMPLEMENTATION

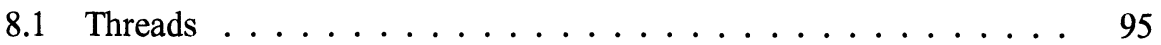

8.2 Representing the Constraint Store . . . . . . . . . . . . . . . 96

8.3 The Abstract Machine . . . . . . . . . . . . . . . . . . . . 98

8.4 Implementation Issues . . . . . . . . . . . . . . . . . . . . . . . . . . . . . . .

8.5 A Realistic Implementation . . . . . . . . . . . . . . . . . . . . . . 102

8.6 Performance Evaluation . . . . . . . . . . . . . . . . 111

8.7 Historical Notes and Related Work . . . . . . . . . . . . . . . 115

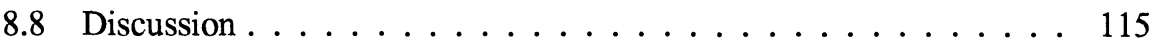

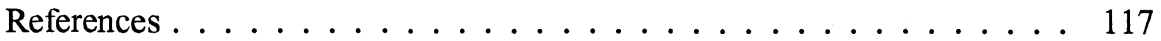

\section{Part III OBJECTS AND CONCURRENCY 119}

9 SYNCHRONIZATION TECHNIQUES 121

9.1 Data-Driven Synchronization . . . . . . . . . . . . . . 121

9.2 Mutual Exclusion . . . . . . . . . . . . . . . . . . . . 122

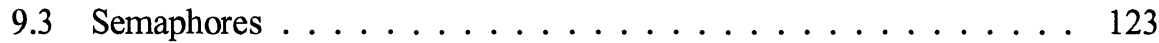

9.4 Bounded Buffer . . . . . . . . . . . . . . . . . . 125

9.5 Readers/Writer . . . . . . . . . . . . . . . . . . . . 127

9.6 Time Behavior . . . . . . . . . . . . . . . . 128

9.7 Locks . . . . . . . . . . . . . . . . . . . . . . . . . . . . . . . . . . . . . . . . . .

9.8 Thread-Reentrant Locks . . . . . . . . . . . . . . . . . . . . . . . . . . . 129

9.9 Objects with Reentrant Locks . . . . . . . . . . . . . . . . . . . . . . . . . . . . . . . . . . . . . . . .

9.10 Inheritance and Concurrency . . . . . . . . . . . . . . 133

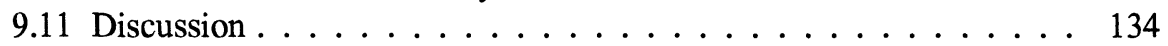

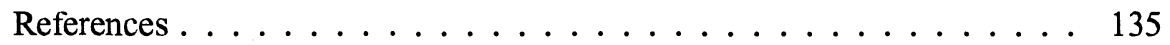

10 ACTIVE OBJECTS 139

10.1 Many-to-One Communication . . . . . . . . . . . . . . . 139

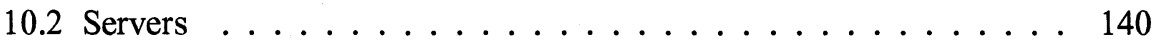

10.3 Case Study: Contract Net Protocol . . . . . . . . . . . . . . . 142

10.4 Performance Analysis . . . . . . . . . . . . . . . . . . . . . . . . . . . . 144

10.5 Discussion . . . . . . . . . . . . . . . . . . . . 145

10.6 Historical Notes and Related Work . . . . . . . . . . . . . 146

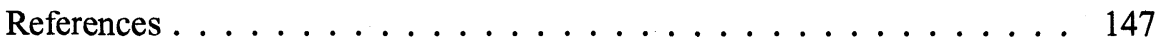

11 ALTERNATIVE CONCURRENCY MODELS

11.1 Fine-Grained Concurrency . . . . . . . . . . . . . . . . 151

11.2 Objects for Fine-Grained Concurrency . . . . . . . . . . . . . 152

11.3 Implicit Concurrency . . . . . . . . . . . . . . . . . . . . 152

11.4 Objects for Implicit Concurrency . . . . . . . . . . . . . . . . . . . . . . . . . . . . . . . . . . .

11.5 Summary and Historical Perspective . . . . . . . . . . . . . . . . . . . . . . . . . . . . . . . . . .

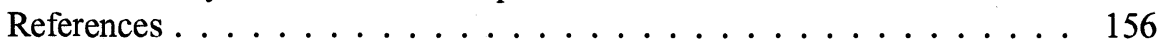


12 A CONCURRENT META-OBJECT PROTOCOL 159

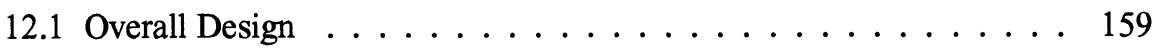

12.2 Object Creation . . . . . . . . . . . . . . . 160

12.3 Method and Object Application . . . . . . . . . . . . . . . . . 162

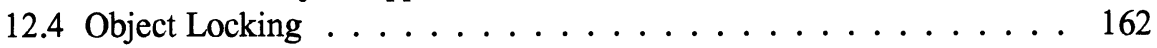

12.5 Discussion and Related Work . . . . . . . . . . . . 165

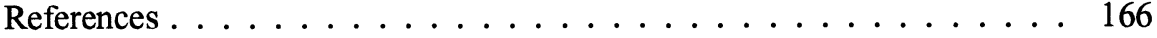

$\begin{array}{ll}\text { INDEX } & 167\end{array}$ 


\section{LIST OF FIGURES}

1.1 Language Design Principles $\ldots \ldots \ldots \ldots \ldots$

1.2 Language Design Cycle . . . . . . . . . . . . . . . . . . 5

1.3 Chapter Dependencies . . . . . . . . . . . . . . . 12

2.1 Control Flow in Procedural Languages (time centered) . . . . . . . 19

2.2 Control Flow in Object-Oriented Languages (time centered) $\ldots \ldots .20$

2.3 Execution of Object Application . . . . . . . . . . . . . 21

2.4 Control Flow in Procedural Languages (state centered) . . . . . . . 24

2.5 Control Flow in Object-Oriented Languages (state centered) . . . . 25

3.1 Syntax of Basic Oz Statements . . . . . . . . . . . . . . . 37

3.2 Value Types in OPM . . . . . . . . . . . . . . . . 41

3.3 Syntax Extension for Records . . . . . . . . . . . . . 42

3.4 Syntax Extension for Cells . . . . . . . . . . . . . . . 44

5.1 Configurations of Sieve of Eratosthenes $\ldots \ldots \ldots \ldots$

6.1 Example of Inheritance Graph . . . . . . . . . . . . . . . . . 74

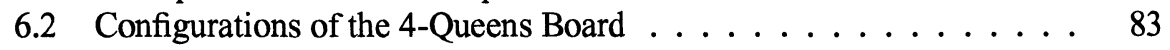

7.1 Structure of an Account Object . . . . . . . . . . . 90

$8.1 \quad$ Life Cycle of Threads . . . . . . . . . . . . . . . . . . 96

8.2 Nodes on the Heap . . . . . . . . . . . . . . . . . . . . . . . 97

8.3 A Closure in the Store . . . . . . . . . . . . . . . . . . . . . 100

8.4 Machine Code implementing Procedure Application . . . . . . . . 100

8.5 Memory Layout of Two Objects of the Same Class . . . . . . . . . 103

8.6 A Special Method Closure . . . . . . . . . . . . . . . 106

9.1 Configurations of Bounded Buffer $\ldots \ldots \ldots \ldots$

10.1 A Simplified Contract Net Protocol . . . . . . . . . . . . . . 143

12.1 The Structure of the Meta-Object Protocol . . . . . . . . . . . . 160 


\section{LIST OF PROGRAMS}

3.1 An Example for Higher-Order Programming . . . . . . . . . . . . 39

3.2 Example for Record Construction . . . . . . . . . . . . . 43

3.3 A Mapping Procedure in $\mathrm{Oz} \ldots \ldots \ldots \ldots$. . . . . . . 47

4.1 A Stateful Procedure . . . . . . . . . . . . . . . . . 54

4.2 Generating Stateful Procedures . . . . . . . . . . . . . . . 54

4.3 Generating Stateful Procedures with Late Binding . . . . . . . . . . 55

4.4 Delegation-based Code Reuse with Passive Objects . . . . . . . . 56

4.5 Processing Messages of Active Objects . . . . . . . . . . 58

4.6 Delegation-base Code Reuse with Active Objects . . . . . . . . 59

5.1 A Simple Account Class . . . . . . . . . . . . . . . . 63

5.2 Conservative Extension through Inheritance . . . . . . . . . 65

5.3 An Account with Fee . . . . . . . . . . . . . . 66

5.4 Sieve of Eratosthenes . . . . . . . . . . . . . . . . . 67

6.1 Use of Object Features in an Account with Fee . . . . . . . . 72

6.2 Using Free Feature and Attribute in Sieve of Eratosthenes . . . . . . 73

6.3 Example for Friends . . . . . . . . . . . . . . . . . 77

6.4 A Parameterized Class for Account with Fee . . . . . . . . . . . . 80

6.5 Calculator . . . . . . . . . . . . . . . . 81

$6.6 \mathrm{~N}-\mathrm{Queens}$ in Small $\mathrm{Oz} \ldots \ldots \ldots \ldots \ldots$

7.1 Overall Structure of Object Library . . . . . . . . . . . . . . 88

7.2 Class Definition . . . . . . . . . . . . . . . . . . 89

7.3 Library Procedure for Object Creation . . . . . . . . . . . . 90

7.4 Library Procedures for State Use . . . . . . . . . . . . . . . . . 92

7.5 Library Procedure for Method Application . . . . . . . . . . . . 93

7.6 Library Procedure for Object Application . . . . . . . . . . 93

9.1 Producer $/$ Consumer . . . . . . . . . . . . . . . . . . . . . 121

9.2 Mutual Exclusion . . . . . . . . . . . . . . . . . . 123

9.3 Semaphore Class . . . . . . . . . . . . . . . . . . . . . . . 124

9.4 Bounded Buffer . . . . . . . . . . . . . . . . . . 125 
9.5 Readers $/$ Writer Problem . . . . . . . . . . . . . . . . . 127

9.6 A Class for Laziness . . . . . . . . . . . . . . . . . . . . . . . . . 129

9.7 A Repeater Class . . . . . . . . . . . . . . . . . . . . . 130

9.8 Lock . . . . . . . . . . . . . . . . . . . . . . . 130

9.9 Mutual Exclusion with Locks . . . . . . . . . . . . . . . . . 130

9.10 Reentrant Locks . . . . . . . . . . . . . . . . . . . . . . . 131

10.1 Expressing Ports with Cells . . . . . . . . . . . . . . . 140

10.2 Creating Servers for Objects . . . . . . . . . . . . . . 141

10.3 Encapsulating Objects in Servers . . . . . . . . . . . . . . 141

10.4 A Server Class . . . . . . . . . . . . . . . . . . . . 142

10.5 Negotiation Protocol in a Transportation Scenario . . . . . . . . 143

11.1 Data-Flow Synchronization for State Manipulation . . . . . . . . 154

11.2 Data-Flow Synchronization for Object Application . . . . . . . . 155

11.3 Data-Flow Synchronization for Object Application (seq. version) . . 155

12.1 A Meta-Class for Object Creation $\ldots \ldots \ldots \ldots \ldots 161$

12.2 A Meta-Class for Active Objects . . . . . . . . . . . . . . 162

12.3 A Meta-Class for Object and Method Application . . . . . . . . 163

12.4 A Meta-Class for Agents . . . . . . . . . . . . . . . 163

12.5 A Meta-Class for Locking . . . . . . . . . . . . . . . . . . 164

12.6 A Meta-Class for Implicit Locking . . . . . . . . . . . . . . . . 164

12.7 A Meta-Class for Hierarchical Locking $\ldots \ldots \ldots \ldots$ 


\section{LIST OF TABLES}

8.1 Languages and Systems used for Performance Comparison . . . . . 112

8.2 Performance Figures for "Sieve of Eratosthenes" . . . . . . . . . . 113

8.3 Performance Figures for "16-Queens" . . . . . . . . . . . . . . 113

8.4 Performance Figures without Arithmetics for "16-Queens" . . . . . . 114

10.1 Performance Figures Passive vs Active Objects $\ldots \ldots \ldots \ldots$ 


\section{FOREWORD}

The Oz Project started in 1991 as an effort to better understand the relationship between existing programming paradigms and to design a unified language supporting constraint programming, concurrency, and object-orientation. Our starting point was Logic Programming, then a thriving research area, from which we took many of the ideas for $\mathrm{Oz}$. Other ideas came from functional programming and concurrency theory.

The goals of the Oz Project were mainly practical, not so much theoretical. Early on, it became clear that we could not reach our goals with a purely declarative language. So we took the best ideas from the declarative paradigms and integrated them with state and explicit control. This integration led to a new, surprisingly simple computation model with amazing expressivity. Having grown up with the dogma of the declarative churches "do not consider the impure," it took some time until we felt comfortable with the integration of non-declarative features.

Our goal to support object-oriented abstractions had a severe impact on the design of Oz. Since objects typically do have state, $\mathrm{Oz}$ had to have state. Since object-oriented abstractions are too complex and too diverse to appear as computational primitives, we needed first-class procedures and lexical scoping to express them. Since objects come with first-class identity and classes come with complex visibility rules, we invented a notion of name that can express these features, among many others. Much inspiration for $\mathrm{Oz}$ came from our attempt to reconstruct objects and classes from a minimal set of general purpose primitives.

Dr. Martin Henz was one of the young researchers with whom I started the $\mathrm{Oz}$ Project. His first contribution was the beautiful name Oz. His most tedious duty was the consolidation of the $\mathrm{Oz}$ syntax from the conflicting wish lists of the project members. His main task, however, was the design and implementation of an object system for the envisioned language. He did a marvelous job. This book, a slight revision of his doctoral thesis, gives a first hand account of "objects in Oz." It will be of great interest to language designers, language implementors, and serious $\mathrm{Oz}$ programmers.

Gert Smolka

Saarbrücken 


\section{PREFACE}

Object-oriented programming had a tremendous impact on the world of software in the last 15 years. Numerous approaches have been proposed to support this successful programming paradigm in other programming language families such as logic and functional programming.

Concurrent constraint programming (ccp) is a recent development in programming language design. Its central contribution is the notion of partial information provided by a shared constraint store. The constraint store serves as communication medium between concurrent threads of control and as vehicle for their synchronization.

In this work, we analyse the possibilities to support object-oriented programming in ccp. Starting from known approaches, we cover various object models and discuss their properties. As model language for the analysis, we use the language Small Oz, a sublanguage of the ccp language $\mathrm{Oz}$. We present a general-purpose object system for Small Oz, describe its implementation and its expressivity for concurrent computation.

The book is written for programming language researchers with interest in programming language aspects of concurrency, object-oriented programming or constraint programming. Programming language implementors will benefit from the rigorous treatment of the efficient implementation of Small $\mathrm{Oz}$. Oz programmers get a firsthand view of the design decisions that lie behind the Oz object system.

From the perspective of ccp, this work intoduces the notion of passive objectsthe central notion of object-oriented programming - to the ccp framework. It builds a bridge from essential ideas of ccp to main-stream programming languages.

From the perspective of object-oriented and functional languages, a central contribution is that partial information as provided by logic variables can be integrated in the framework of both of these paradigms in a straightforward manner. From this intergration, concurrent objects benefit through simple and elegant synchronization techniques.

The language $\mathrm{Oz}$ was designed in the Programming Systems Lab, Saarbrücken, Germany, as an attempt to integrate essential aspects of the paradigms of constraint (logic), object-oriented and functional programming. This book gives account of this integration from the perspective of (concurrent) object-oriented programming. It is based on the author's doctoral thesis "Objects in Oz," accepted by the Universität des Saarlandes in June 1997. 


\section{ACKNOWLEDGEMENTS}

Research is a dynamic and interactive activity which thrives in an environment that fosters exchange of ideas and intense collaboration between researchers. I found such an environment in the Programming Systems Lab in Saarbrücken. It was the cooperation with the enthusiastic, knowledgeable and cooperative researchers of this lab that led to the results reported in this monograph. To say that without them this work would not have been possible would miss the point. This is their work as well as it is mine.

For my doctoral thesis work on which this monograph is based, I was fortunate to be supervised by Gert Smolka. His work on $\mathrm{Oz}$ laid the base for this monograph. His tireless striving for simplicity as a chief goal of scientific endeavor was truly inspiring. Ralf Scheidhauer implemented the emulator support and contributed several ideas to the implementation of the object system that I describe. Christian Schulte contributed countless ideas to the design of Objects in $\mathrm{Oz}$, and Jörg Würtz helped lay the base for its initial design. Christian Schulte and Konstantin Popov were the first object-oriented programmers in $\mathrm{Oz}$ and shared their programming experience with me. Martin Müller and Michael Mehl contributed concurrent programming examples. Michael Mehl helped with comments and suggestions and shared his experience with object-oriented programming. Martin Müller and Joachim Niehren shared their knowledge concerning a few fundamental aspects that I discuss in passing. Denys Duchier contributed an elegant syntactic detail. I thank Seif Haridi for several fruitful discussions on the design of object systems. Leif Kornstaedt, Kelly Reedy, Ralf Scheidhauer, Christian Schulte, Gert Smolka and Joachim Walser commented on earlier versions of this work. The following people provided advice and assistance for the performance measurements in Section 8.6: Hubert Baumeister, Seif Haridi, Michael Mehl, Tobias Müller, Jérôme Vouillon, Peter Van Roy, and Joachim Walser.

The work that led to this book was carried out at the Programming Systems Lab in Saarbrücken from January 1992 to June 1997. From January 1992 to March 1996, I was funded by DFKI (German Research Center for Artificial Intelligence) under contract ITW 9105 with the Bundesminister für Bildung, Wissenschaft, Forschung und Technologie, and from April 1996 to June 1997 by the Universität des Saarlandes, Saarbrücken, Germany, under the Special Research Division "Resource-Adaptive Cognitive Processes", Sonderforschungsbereich 318.

Martin Henz

Singapore 